\title{
L'édition intégrale en ligne des manuscrits de Madame Bovary
}

Yvan Leclerc

\section{OpenEdition}

12 Journals

Édition électronique

URL : http://journals.openedition.org/recherchestravaux/111

DOI : 10.4000/recherchestravaux.111

ISSN : 1969-6434

Éditeur

UGA Éditions/Université Grenoble Alpes

\section{Édition imprimée}

Date de publication : 15 juin 2008

Pagination : 229-240

ISBN : 978-2-84310-125-0

ISSN : 0151-1874

Référence électronique

Yvan Leclerc, "L'édition intégrale en ligne des manuscrits de Madame Bovary », Recherches \& Travaux [En ligne], 72 | 2008, mis en ligne le 15 décembre 2009, consulté le 03 février 2021. URL : http:// journals.openedition.org/recherchestravaux/111; DOI : https://doi.org/10.4000/recherchestravaux. 111

(c) Recherches \& Travaux 


\section{L'édition intégrale en ligne des manuscrits de Madame Bovary}

L'édition intégrale en ligne des manuscrits de Madame Bovary est une entreprise qui a comporté plusieurs phases : la numérisation de l'intégralité du manuscrit par la Bibliothèque municipale de Rouen (dix mois, en 2003), la transcription de tous les folios par des bénévoles encadrés par Danielle Girard (deux ans et demi, de mars 2004 à septembre 2006) et la construction du site qui devait être ouvert à la fin de l'année 2007, pour le cent cinquantenaire du roman, mais a dû être légèrement retardé à cause de problèmes informatiques. L'inauguration du site marque un terme symbolique, mais le chantier ne sera jamais tout à fait terminé. Une édition sur papier est considérée comme arrêtée avec ce qui s'appelle fort justement le «bon à tirer» puis avec l'«achevé d'imprimer», mais l'édition en ligne, surtout d'un manuscrit, n'a pas de fin, pour toutes sortes de raisons : un illisible trouvera un jour son déchiffreur, une faute de transcription son correcteur, un document nouveau de genèse apparaittra; et nos entreprises, si exhaustives puissent-elles paraittre dans leur complétude et leur autonomie (ici, l'intégralité des manuscrits de Madame Bovary), prendront place à long terme, par interconnexions, dans des ensembles plus vastes (les dossiers de genèse de Flaubert, une base HyperFlaubert regroupant toutes les données numérisées). Le produit fini n'est pas encore livré au public; au moins Danielle Girard et moi avons-nous un peu plus d'expérience, un peu plus de recul, et c'est dans cette distance, à la fois théorique et pratique, que nous voudrions aborder les apports et les limites de cette publication numérique, envisagés aux points de vue matériel, intellectuel, institutionnel et médiatique. 


\section{Corpus matériel}

Nous tomberons d'accord pour dire que la publication en ligne est la mieux adaptée à l'édition des corpus volumineux et discontinus. Linéaire et relié, le livre est limité en nombre de pages pour des raisons économiques et pratiques. Par ses possibilités d'extension, de combinaisons et de configurations, le numérique est particulièrement adapté à l'édition des corpus manuscrits.

La nécessité de dématérialiser s'accroît avec le volume des matériaux primaires.

Il y a cinquante ans, un volume de cinq cents pages pouvait contenir une «nouvelle version» de Madame Bovary, telle que Jean Pommier et Gabrielle Leleu l'avaient reconstituée en composant un texte factice truffé de passages supprimés ${ }^{1}$. Le papier peut également accueillir les transcriptions intégrales d'une partie des brouillons : le chapitre des Comices² ou celui de l'Opéra'. Les soixante et une pages des Plans et scénarios tenaient également dans un livre4 : nous étions alors en I995, et il avait été possible de proposer aux acheteurs du volume une disquette comportant une version hypertexte, que Daniel Ferrer, son concepteur, avait intitulée "Hyperscénario pour Madame Bovary». Cette disquette était chère ( 75 francs ajoutés aux 265 francs du livre); elle ne fonctionnait que sur Macintosh, et les commandes chez l'éditeur ont dû se compter à l'unité.

Mais lorsqu'on vise la complétude d'un corpus, non pas à deux ni à trois mais à quatre chiffres, 4500 pages environ pour le dossier manuscrit de Madame Bovary, le papier s'autodétruit. Le cas de Flaubert est exemplaire par la proportion, sans doute l'une des plus fortes dans l'histoire littéraire, entre le manuscrit et l'imprimé. La métaphore de l'iceberg s'impose par la justesse du rapport entre les neuf dixièmes immergés et le dixième visible.

Sur le plan du corpus, l'édition numérique apparaît sans limite, à l'exception de celles qui sont liées à la réception et à l'usage. On peut lire mot à mot le

I. Madame Bovary, nouvelle version précédée des scénarios inédits, par J. Pommier et G. Leleu, José Corti, I 949.

2. $2^{\mathrm{e}}$ partie, chap. 8. Voir Gustave Flaubert, Madame Bovary (Extrait). Les Comices agricoles de Gustave Flaubert; transcription intégrale et genèse dans le manuscrit G 223 par J. Goldin, Genève, Droz, 1984 .

3. $2^{\mathrm{e}}$ partie, chap. I 5 . Voir M. MacNamara, La Textualisation de Madame Bovary, Amsterdam et New York, Rodopi, 2003.

4. Plans et scénarios de Madame Bovary, transcription du manuscrit et présentation par Y. Leclerc. Répertoire des manuscrits de Flaubert et Bibliographie génétique de Flaubert, O. de Guidis. Hyperscénario pour Madame Bovary: un outil de navigation hypertextuel, D. Ferrer, CNRS éditions / Zulma, «Manuscrits», I 995 . 
roman intitulé Madame Bovary; on peut encore lire une «nouvelle version» gonflée du même roman, mais on ne peut pas lire 4500 pages de manuscrits, même rendues accessibles par leur transcription. La lecture reste encore attachée au papier, pour le confort des yeux et par les habitudes mentales héritées de plusieurs millénaires de «linéarité du signifiant», comme on disait jadis.

Comment appréhender des milliers de pages? Les trois modes d'accès au manuscrit retenus pour la navigation dans le site final permettront de «Feuilleter» (dans l'ordre matériel de la conservation), de «Consulter» (par séquences génétiques) et de «Rechercher» (à l'aide d'un moteur). Ce sont toutes des opérations discontinues qui ne forment pas un lecteur mais une nouvelle catégorie d'usagers de la chose écrite dont nous ne connaissons pas encore le profil. En multipliant le nombre de pages de Madame Bovary par dix ou vingt, nous n'avons pas multiplié notre temps disponible de lecture ou de travail : nous avons mis à disposition des matériaux pour construire des parcours personnels de lecture. Au risque de créer, chez les lectrices et les lecteurs confrontés à l'infini des possibles, un nouveau type de bovarysme...

L'ensemble du dossier génétique se caractérise par son hétérogénéité. Les types de documents ne sont pas de même nature : il y a de grandes différences de structure entre le manuscrit autographe définitif et le texte du copiste d'une part, tous les deux textualisés et foliotés, et permettant la lecture syntagmatique en continu, et d'autre part les pages de brouillons, inégalement textualisées et présentant des solutions de continuité entre les folios d'une même strate génétique et des différences d'amplitude entre les états superposés à la verticale d'un même fragment de texte. Même avec ces décalages verticaux et ces ruptures horizontales, il est possible de faire figurer tous les folios des brouillons et des deux manuscrits terminaux dans un même tableau génétique étalonné page par page sur la base du manuscrit autographe définitif.

Il en va différemment pour les plans et les scénarios : 6I pages programment 4500 pages de brouillons. Les unités n'ont plus de commune mesure : le premier plan général connu couvre en une page et demie tout le roman à venir; la scène du fiacre «sort» de quelques mots placés en marge d'un scénario sectoriel $\left(\mathrm{f}^{\circ} 33 \mathrm{r}^{\circ}\right)$. En raison de cette différence d'échelle, on a renoncé à faire figurer ces plans et scénarios dans le tableau génétique général. Dans la rubrique «Consulter», on les trouvera dans un tableau génétique à part (ils sont aussi accessibles par la rubrique «Feuilleter», et pris en compte dans les résultats de l'interrogation par le moteur de recherche). Pour intégrer la phase programmatique dans le tableau génétique global du dossier de genèse, tous documents confondus, il faudrait imaginer une navigation dont l'unité de référence ne serait plus le folio, mais des unités de dimensions très variables : un mot du scénario, tel que «fiacre», pouvant ouvrir sur tout un épisode comportant 
plusieurs séquences, constituées d'un nombre considérable de folios. Encore notre exemple n'est-il pas significatif, dans la mesure où le mot «fiacre» est limité dans Madame Bovary à la scène du même nom : pour faire sortir ensemble le mot isolé du scénario et les séquences rédactionnelles où il apparait, il suffit de taper «fiacre» dans le moteur de recherche. Mais cette possibilité ne se présente plus si l'épisode développé dans les brouillons est désigné au stade des plans et scénarios par un mot qui ne se trouve pas ailleurs : pensons à la scène de la «baisade», ainsi nommée quand Flaubert écrit «pour lui», mais qui n’apparait évidemment pas dans le roman imprimé.

Comme on le sait, la documentation pour Madame Bovary est réduite à peu d'éléments externes. Rien de commun avec le texte qui précède, La Tentation de saint Antoine de I 849, et avec ceux qui suivent, dans lesquels les lectures et les notes ont un poids considérable. Pour Madame Bovary, nous nous trouvons devant deux cas de figures : ou bien les notes ont été prises dans la continuité du manuscrit (par exemple pour l'épisode du pied-bot), ou bien elles se trouvent hors dossier de Madame Bovary. Pour celles-ci, se pose comme pour les plans et scénarios le problème de l'intégration à la navigation, non plus en raison de la différence d'échelle mais à cause de leur statut génétique particulier. Dans l'état actuel du site, nous leur avons réservé une place dans la rubrique «Feuilleter», en leur ménageant la même autonomie que les plans et scénarios, sans lien avec le tableau génétique général. On aurait pu évidemment ajouter un étage supplémentaire à ce tableau pour les y faire figurer, mais avec l'inconvénient d'empiler à la verticale des couches génétiques disparates brouillant la lisibilité, et avec la difficulté de placer certaines notes à un endroit pertinent du déroulement du roman : les notes sur l'arsenic peuvent trouver un point d'ancrage précis, mais où situer les Mémoires de Madame Ludovica qui racontent les déboires financiers d'une femme? Pour l'édition en ligne des brouillons du premier chapitre de la troisième partie de L'Éducation sentimentale, Tony Williams a adopté une solution originale : il donne à part la transcription intégrale et en continu des notes documentaires et il indique dans les transcriptions les liens vers les notes ponctuelles par des «points chauds» (appels en rouge)'. On peut donc remonter des états manuscrits vers la source documentaire, mais non pas faire le trajet inverse, de la note vers sa mise en texte, sans doute parce que la première opération renvoie directement d'une pluralité de fichiers à un signet sur un fichier unique, alors que pour aller de telle note à tous les fichiers qui en portent la trace, il aurait fallu passer par une page intermédiaire de distribution.

De proche en proche et les cercles concentriques s'élargissant, on voit bien ce qu'Internet permet de rêver, à défaut de pouvoir encore le réaliser :

5. «L'histoire en question», http://www.hull.ac.uk/hitm/. 
des sommes exhaustives. Comme au temps des humanistes et des encyclopédistes, nous poursuivons la réalisation d'ensembles complets et hiérarchisés. Chacun dans son domaine rêve d'un Hyper quelque chose ou quelqu'un. Nous-même parlions d'un HyperFlaubert dès 2002, au colloque de Lisieux ${ }^{6}$.

L'extension hypertexte à partir du manuscrit de Madame Bovary pourra se faire en reliant le tableau génétique au calendrier de la genèse, établi sur la correspondance, qui, outre des dates, présente un discours d'accompagnement esthétique, essentiellement dans les lettres adressées à Louise Colet. L'échange avec Louis Bouilhet passait plus dans la conversation orale que par la voie postale, mais certaines de leurs lettres, dans les deux sens, jouent un rôle documentaire dans la genèse, en particulier pour la description de l'Aveugle. Pourrait intervenir également dans cet HyperBovary la bibliothèque de Flaubert et, sur une orbite plus éloignée, les différentes éditions originales du roman, les comptes rendus auxquels il a donné lieu, les traductions, les illustrations, les adaptations, les articles et ouvrages critiques, etc., jusqu'aux travaux suscités par les transcriptions installées sur le site.

\section{Aspects intellectuels}

Ces considérations matérielles sur le corpus conduisent à envisager les aspects intellectuels de l'édition numérique. Un manuscrit est en effet un objet à deux faces, physique et mentale, inséparables comme le signifiant et le signifié de Saussure. Dans nos entreprises de numérisation, les tâches se répartissent en fonction de ces deux aspects : les conservateurs s'occupent du support physique et les chercheurs apportent la «valeur intellectuelle» ajoutée, avec évidemment des interférences possibles, puisque Pierre-Yves Cachard, conservateur à la Bibliothèque municipale de Rouen responsable de la numérisation, a lui-même assuré la transcription d'une séquence du roman. En ce qui concerne l'édition numérique de Madame Bovary, l'apport du Centre Flaubert concerne trois domaines.

Premier apport: le classement génétique intégral du dossier. L'ordre matériel de conservation, établi par les premiers bibliothécaires en charge du manuscrit, garde son utilité si l'on veut accéder par la rubrique «Feuilleter» à un folio dont on connait le numéro ou aux volumes physiques des manuscrits, tels qu'ils sont reliés aujourd'hui. Mais la consultation et la recherche nécessitent évidemment un classement chronologique fin de l'ensemble du dossier. Pour

6. «Les études françaises valorisées par les nouvelles technologies d’information et de communication (littérature - Internet - bibliothèque)», Bibliothèque municipale de Lisieux, 27-28 mai 2002. 
Madame Bovary, le classement statique a été établi par Hisaki Sawasaki et le classement génétique menée à son terme par Marie Durel ${ }^{8}$. Claudine GothotMersch a par ailleurs eu l'extrême amabilité de nous transmettre le classement préparatoire qu'elle avait effectué en vue de sa thèse sur la Genèse de Madame Bovary ${ }^{9}$. Classer page par page représente un travail de plusieurs années, qui exige la résistance du coureur de fond, la patience de Pénélope, la minutie d'un entomologiste et la détermination d'un bâtisseur de pyramide. L'opération sur les manuscrits de Madame Bovary a été effectuée dans le cadre d'une thèse, financée par une bourse doctorale du Conseil régional de HauteNormandie. La question se posera pour les autres corpus à classer : comment mener à bien cette tâche? Car à part Madame Bovary, seul le manuscrit intégral de Trois contes a été classé par Giovanni Bonaccorso ${ }^{10}$, et certains chapitres de Bouvard et Pécuchet (en particulier le chapitre V, classé et transcrit par Stéphanie Dord-Crousléri ${ }^{11}$.

Deuxième apport: la transcription intégrale des manuscrits. L'écriture de Flaubert n'est pas immédiatement déchiffrable : elle se situe dans une moyenne sur l'échelle de la lisibilité : plus difficile que celle de Zola et Maupassant, qui peuvent le plus souvent se passer de transcription, mais plus facile que celle de Stendhal, à cette différence près toutefois que Stendhal rature peu, pour les raisons esthético-morales que l'on sait, alors que les ratures flaubertiennes nous obligent trop souvent à déclarer forfait et à recourir à un «illis.». Notre transcription ne se veut pas «diplomatique»; elle se contente d'être "analogique», selon le terme employé par Danielle Girard : la présence de l'image nous donne en effet un peu de liberté, dans la mesure où notre transcription ne se substitue pas, comme souvent sur le papier, à un manuscrit absent, mais aide à le déchiffrer en lecture directe. Indispensable pour lire le manuscrit, la transcription l'est aussi pour permettre au moteur de recherche d'effectuer les requêtes demandées.

Troisième apport: la mise au point de la navigation et des fonctionnalités du moteur de recherche, en liaison avec les autres partenaires. Danielle Girard et

7. Voir la présentation de son travail sur le site des Manuscrits de Madame Bovary : http://flaubert.univ-rouen.fr/o2manus/I77_detachement/o_presi 77.htm.

8. M. Durel, «Classement et analyse des brouillons de Madame Bovary de Gustave Flaubert», thèse de Doctorat, Université de Rouen, 2000.

9. Cl. Gothot-Mersch, thèse de Doctorat soutenue en I963, La genèse de Madame Bovary, José Corti, I966, réimprimée par Slatkine en I 980.

ı. Corpus flaubertianum II, Hérodias, t. I, édition diplomatique et génétique des manuscrits, par G. Bonaccorso et ses collaborateurs, t. I, Nizet, I 99 I.

I I. «Étude génétique et critique du chapitre V de Bowvard et Pécuchet de Gustave Flaubert», thèse de Doctorat (nouveau régime) sous la direction de J. Neefs, Université Paris VIII Saint-Denis, I 998. 
moi avons insisté sur la nécessité de concilier les exigences d'utilisateurs très divers, depuis les lycéens qui ont besoin, au bout de cinquante minutes de cours, de sauvegarder des résultats de recherche dans un "panier» pour les exploiter ensuite hors connexion, jusqu'aux chercheurs "pointus» qui voudront faire porter leur requête sur des secteurs bien délimités du corpus, et qui attendront des résultats exhaustifs.

Ces apports d'ordre intellectuel (classer, transcrire, consulter et rechercher) n'ont eu lieu que dans le cadre et dans la perspective de l'édition numérique : consulter et rechercher, en ce sens, demandent bien évidemment cette forme nouvelle d'édition, mais aussi classer et transcrire, opérations qui n'ont fort heureusement pas attendu le numérique, mais qui s'effectuent aujourd'hui selon d'autres modalités. Par exemple, le classement intégral des 4500 pages a été poussé jusqu'à la correspondance ligne à ligne entre les folios pour permettre la constitution d'une base de données tenant compte de l'amplitude des segments développés dans chaque folio, ce qui eût été un luxe superflu pour une mise en ordre destinée à la fixité d'un livre. Il en va de même pour la transcription intégrale : l'éventualité d'une publication sur papier pour un corpus aussi volumineux étant quasi nulle, il ne se serait trouvé personne pour commencer ce travail de copiste, même pas Bouvard et Pécuchet, qui copient comme on sait des citations, et non des pages entières.

Pour des travaux de cette ampleur, ce que les scientifiques des sciences dures pratiquent depuis longtemps dans le cadre des laboratoires s'impose désormais à nous : la collaboration ${ }^{12}$. Elle est rendue possible par les échanges instantanés entre chercheurs distants. Auparavant, les «littéraires» étaient seuls face à leur sujet : voir le cas exemplaire de Sartre, qui avait un «compte à régler avec Flaubert». On a toujours un compte à régler avec Flaubert, mais on le règle désormais collectivement. La chance que nous avons eue à Rouen pour ce projet Madame Bovary, c'est de constituer une vraie équipe de travail, avec un petit noyau dur "présentiel», comme on dit aujourd'hui, fortement soudé par des relations de confiance et d'amitié, hors volonté de pouvoir ou désir de reconnaissance institutionnelle, et une vaste équipe virtuelle à la dimension et à la hauteur du projet. Les équipes qui n'en ont que le nom et pas le mode de fonctionnement sont condamnées à se dissoudre dans les grands projets numériques.

Le travail d'équipe a eu pour effet de poser des questions d'ordre juridique. Elles existent évidemment pour tout mode d'édition, mais elles deviennent plus aiguës avec le numérique, nous a-t-il semblé, en raison de deux facteurs

I 2. On évitera d'utiliser l'expression «annotation collaborative»: dans le vocabulaire de l'édition : «annotation» a un sens bien précis, dont nous ne devons pas nous laisser déposséder par les informaticiens. 
apparemment opposés : d'une part le laxisme général lié à la circulation massive et éphémère d'informations qui ont tendance à s'anonymer, et d'autre part la visibilité universelle de la publication sur Internet qui rend les collaborateurs légitimement vigilants. Par exemple, une chercheuse s'est manifestée au début du projet pour revendiquer un droit de propriété; nous qui sommes habitués à ce que le droit appelle «la libre circulation des idées», au partage du savoir et à l'utilisation des résultats d'une recherche, à la seule condition de la citer, nous avons découvert qu'une thèse avait été déposée à la Société des auteurs - ce qui constitue à ma connaissance un précédent -; nous avons appris qu'une base de données est protégée par les droits de propriété intellectuelle, même quand son auteur a bénéficié d'une bourse dans le cadre d'un laboratoire pour la mettre en œuvre. Le problème des droits s'est également posé à nous avec les chapitres des Comices et de l'Opéra : nous nous sommes bien évidemment aidés des transcriptions existantes, en signalant notre dette à l'égard de Jeanne Goldin et de Matthew MacNamara, mais nous avons procédé à une nouvelle saisie, selon un autre code, et en collationnant nos transcriptions sur l'autographe, corrigeant au passage quelques inévitables coquilles ou fautes de lecture. La Bibliothèque municipale de Rouen, de son côté, a demandé aux transcripteurs de s'engager à n'utiliser les fichiers haute définition qui leur étaient transmis que pour un usage privé, dans le cadre de leur participation au projet, sans autorisation de les céder ni de les reproduire. Le Centre Flaubert a mis au point une formule pour répondre aux demandes de citation ou de reproduction :

Toute réutilisation des transcriptions à des fins privées, à des fins d'enseignement ou de recherche scientifique est autorisée, sous réserve d'indiquer cette référence :

«Transcription(s) réalisée(s) pour l'édition génétique des manuscrits de Madame Bovary (site Flaubert, Université de Rouen).»

Pour toute publication, demander l'autorisation du Centre Flaubert.

\section{Partenariats institutionnels}

Des entreprises comme les nôtres supposent des montages institutionnels et financiers complexes. Le plus souvent seront associés un organisme de conservation (pour nous la Bibliothèque municipale de Rouen qui possède la quasi-totalité du dossier manuscrit de Madame Bovary, à l'exclusion de quelques notes), et une institution d'enseignement et de recherche (l'Université de Rouen), qui a signé avec la Bibliothèque en 2003 des conventions de coopération et de recherche. Ces conventions sont nécessaires pour définir les droits et les devoirs de chacun, mais aussi pour se protéger contre d'éventuelles OPA 
inamicales qui peuvent toujours se déclarer de la part d'autres groupes de recherche, tant sont grandes les convoitises suscitées par des entreprises internationales et mondialement visibles. Il y a là de réels enjeux de pouvoir symbolique en termes d'image, de reconnaissance institutionnelle, d'expertise, d'autorité et de notoriété.

Ces attelages institutionnels sont indispensables mais aussi improbables, et sources de tensions, si l'on n'y prend pas garde. Mettre ensemble des conservateurs, des chercheurs en littérature, et des informaticiens, doit aboutir à ce que les technocrates appellent une «synergie» par complémentarité d'intérêts et de compétences autour d'un même objet. Mais nous avons, les uns et les autres, des pratiques, des langages et des buts différents, et chacun persévère dans son être. Les conservateurs conservent: en numérisant, ils obéissent à un double objectif de préservation et de mise à disposition du public. Même s'ils ne sont pas insensibles à la valeur intellectuelle ajoutée, ils privilégient le trésor patrimonial qu'ils ont en dépôt. Si bien que l'installation sur le site des notes documentaires de Madame Bovary, conservées à la Fondation Bodmer, à Genève, n'est pas jugée prioritaire par la Bibliothèque municipale, dans la mesure où elles n'appartiennent pas à son fonds. Les chercheurs cherchent: ce qui constitue un point d'arrivée par les conservateurs est un point de départ pour eux. Le matériau primaire doit être traité, construit intellectuellement pour être utilisable, en vue de travaux futurs. Ils s'intéressent à l'ensemble du dossier de genèse, quel que soit son lieu physique de conservation. Les informaticiens informatisent, sans toujours comprendre les besoins de la recherche, et parfois aux dépens du contenu, que l'informatique doit apprendre à servir silencieusement au lieu de s'en servir comme faire-valoir.

La pluralité des partenariats va de pair avec la multiplicité des financements. Les projets numériques reçoivent généralement de l'argent de tous les étages administratifs : ville, département, région, État. Un ami parisien, et baudelairien, me disait récemment que Flaubert avait bien de la chance d'être né en province. On pourrait en dire autant pour Stendhal. Eux-mêmes n'auraient pas été très enclins à reconnaitre cette chance, mais les chercheurs grenoblois ou rouennais profitent de cette situation. Ledit ami me disait que la Ville de Paris ne donnait jamais rien pour Baudelaire. En revanche, les institutions locales mettent la main au porte-monnaie à chaque fois qu'on les sollicite pour leurs grands hommes. Le Conseil régional de Haute-Normandie est ainsi le principal financeur de la recherche universitaire en Lettres, sous forme de bourses doctorales, d'aides à projets dans le cadre de l'axe «Patrimoine» de l'IRSHS (Institut de recherche en sciences humaines et sociales), de subventions pour les éditions ou les colloques. 
La question financière concernant les éditions numériques pourrait se résumer à un paradoxe : c'est tellement cher que ça ne peut être que gratuit. Nous sommes un service public aidé par des finances publiques, valorisant un bien patrimonial public au service du «grand public». Nous avions un instant, dans la phase préparatoire, en 2000, envisagé de produire un CD-ROM commercialisé, puis d'ouvrir un site payant. Mais nous avons rapidement abandonné ces idées mercantiles pour un projet tout entier fondé sur l'idée du service public et sur le bénévolat des transcripteurs, et dont les résultats seront en libre accès (la Bibliothèque municipale se réservant la commercialisation des images haute définition des manuscrits). Les projets numériques trouvent encore des opportunités d'aides publiques importantes. Mais ces crédits publics, qui ne seront pas relayés dans le même esprit par des financements privés, viendront à se raréfier, en raison de la banalisation de ces réalisations. Nous faisons encore figures de pionniers, mais plus pour longtemps.

Les partenaires ont des objectifs différents; ils ont aussi des conceptions différentes des publics. Internet permet aux chercheurs de s'adresser à un «lectorat» qui dépasse de beaucoup celui des spécialistes. L'édition savante sur papier, génétique en particulier, était faite par des chercheurs pour des chercheurs. On était entre soi, on s'adressait à ses pairs. Le CD-ROM sur Les Caves $d u$ Vatican $^{13}$, dont la diffusion est restée très confidentielle, faisait l'objet d'un compte rendu dans la revue Genesis, pas plus. Mais en changeant de mode et donc d'échelle de diffusion, on s'adresse à tout public. Ce singulier collectif unifie artificiellement une réalité multiple et impossible à saisir : il y a des publics pour nos éditions, qui s'échelonnent des non-spécialistes aux spécialistes, des amoureux fervents aux savants austères, des curieux (les «badauds du Web», selon l'expression de Françoise Legendre, directrice des bibliothèques municipales de Rouen à l'époque de la conception du projet Bovary) aux chercheurs, des lycéens aux universitaires, des voyeurs du patrimoine aux fétichistes des traces graphiques. Nous avons donc à concilier des impératifs contradictoires et à satisfaire des besoins et des attentes différentes, que nous ne savons même pas formuler. Nous aurons réussi si tous les publics y trouvent leur compte.

\section{La communication}

Dans le même temps que nous découvrons les à-côtés juridiques et institutionnels des grands travaux littéraires numériques, nous sommes confrontés à un aspect nouveau : la communication avec les médias, pratique à

I 3. Les Caves du Vatican, édition génétique. CD-ROM, Gallimard, 200 I. 
laquelle nous sommes peu préparés. Jusqu'à présent, nos activités de chercheurs ne sortaient guère d'un cercle restreint de spécialistes : nous parlons devant des pairs, que sont aussi nos évaluateurs et nos critiques, dans des revues spécialisées qui fonctionnent en circuit fermé. En opérant un changement d'échelle et en sortant de petit monde des chercheurs, Internet modifie la règle du jeu en nous exposant à des médiateurs redoutables : les journalistes. Nous avons pu mesurer l'«impact» (pour parler comme les journalistes) de l'article de trois pages paru dans Libération les 5-6 mars 2005 : à ce stade du projet, il a suscité quelques vocations supplémentaires de transcripteurs. Mais cette communication d'un nouveau type présente des inconvénients qui tiennent moins à la personne des journalistes, plutôt attentifs et bienveillants, qu'à leur façon de rendre compte d'une entreprise collective et d'une édition sur Internet. Dans les années à venir, les journalistes en charge de la littérature seront sans doute plus expérimentés : on ne passe pas facilement de la recension d'un livre le plus souvent à auteur unique au compte rendu d'une œuvre numérique réalisée avec de multiples collaborations. À chaque article de la presse nationale ou régionale, nous éprouvons des sentiments mêlés : le plaisir de la reconnaissance, certes, par exemple quand Le Monde des Liures consacre une demi-page à notre travail, le 20 octobre 2006, mais aussi le malaise de voir la complexité d'une entreprise «innovante» traitée avec des notions anciennes, et la gêne de constater que bien souvent les noms des responsables et des organismes impliqués ont disparu. Cette négligence peut paraittre anecdotique, mais elle atteint pourtant le cœur du dispositif. Par expérience, nous savons qu'une personne ou qu'une institution non citée exprimera son mécontentement, d'autant plus que ces institutions attendent de nos grands travaux un «retour sur investissement» en termes de «valorisation d'image», pour emprunter leur vocabulaire. Nos partenaires ne sont pas des philanthropes : leur politique culturelle se confond avec la culture de leur politique. Ils attendent du soutien qu'ils apportent aux projets numériques une visibilité aux dimensions d'Internet.

L'idée même d'une publication comme celle de l'intégralité des manuscrits de Madame Bovary n'a pu se former qu'avec l'apparition d'un nouveau support. Il est vrai que nous avons la chance de posséder là un roman mondialement connu, dont il est même question dans les premiers épisodes de Desperate Housewives. La notoriété universelle du roman a été déterminante dans le choix du sujet, dans l'obtention des crédits, dans la constitution de l'équipe de transcripteurs et dans l'adresse à un public sans frontières. La donne aurait été différente si l'on avait eu affaire à Salammbô ou à La Tentation de saint Antoine, textes plus confidentiels. Une fois désigné l'objet légitime, Internet permet 
d'étendre indéfiniment les dimensions du corpus et du public visé. À l'horizon de l'HyperBovary se dessine un ToutFlaubert. Mais on perçoit immédiatement les limites qualitatives d'un corpus sans limites quantitatives, hanté par le fantasme d'une totalité positiviste à laquelle il manquera toujours un élément, par le délire de la collection aux dépens du plaisir du sens, et par la collecte des traces en attente d'une interprétation globale, qui ne viendra pas. $\mathrm{Si}$ le corpus est extensible, le temps imparti à chacun, concepteur, réalisateur ou utilisateur, ne l'est pas. Et comment assurer le repérage et la lisibilité dans un Hypermarché textuel où tout sera relié à tout, et réciproquement? Au moins les encyclopédistes du XVIII et du XIX ${ }^{e}$ siècles avaient-ils pour s'y reconnaitre l'ordre uniforme de l'alphabet, pauvre mais efficace. Nous ne sommes pas sûrs de trouver pour nos dossiers complexes des mises en ordre d'une telle simplicité universelle. 